\title{
Three Dimensional Simulations of Hypersonic MHD Flow Control
}

\author{
Nicholas J. Bisek* and Iain D. Boyd ${ }^{\dagger}$ \\ Department of Aerospace Engineering, University of Michigan, Ann Arbor, MI, 48109, USA \\ and \\ Jonathan Poggie $\ddagger$ \\ Air Force Research Laboratory, Computational Sciences Branch, WPAFB, OH, 45433, USA
}

\begin{abstract}
A computational study of hypersonic air flow over a three-dimensional blunt leading edge is carried out to investigate the energy deposition due to an arc discharge along the surface. The discharge is the result of an imposed potential difference between two flush-mounted electrodes. The energy deposition is the result of Joule heating, which is included in the three-dimensional fluid solver along with the magnetic force. These magnetohydrodynamic effects are determined by assuming the current continuity equation for low magnetic Reynolds number flow, and computed using a parallelized finite-volume method. In addition, several electrical conductivity models, including an eleven dimensional surrogate model of solutions to Boltzmann's equation, are explored. It is shown that the computed electric field and subsequent current density field are directly dependent on the electrical conductivity model selected.
\end{abstract}

\section{Nomenclature}

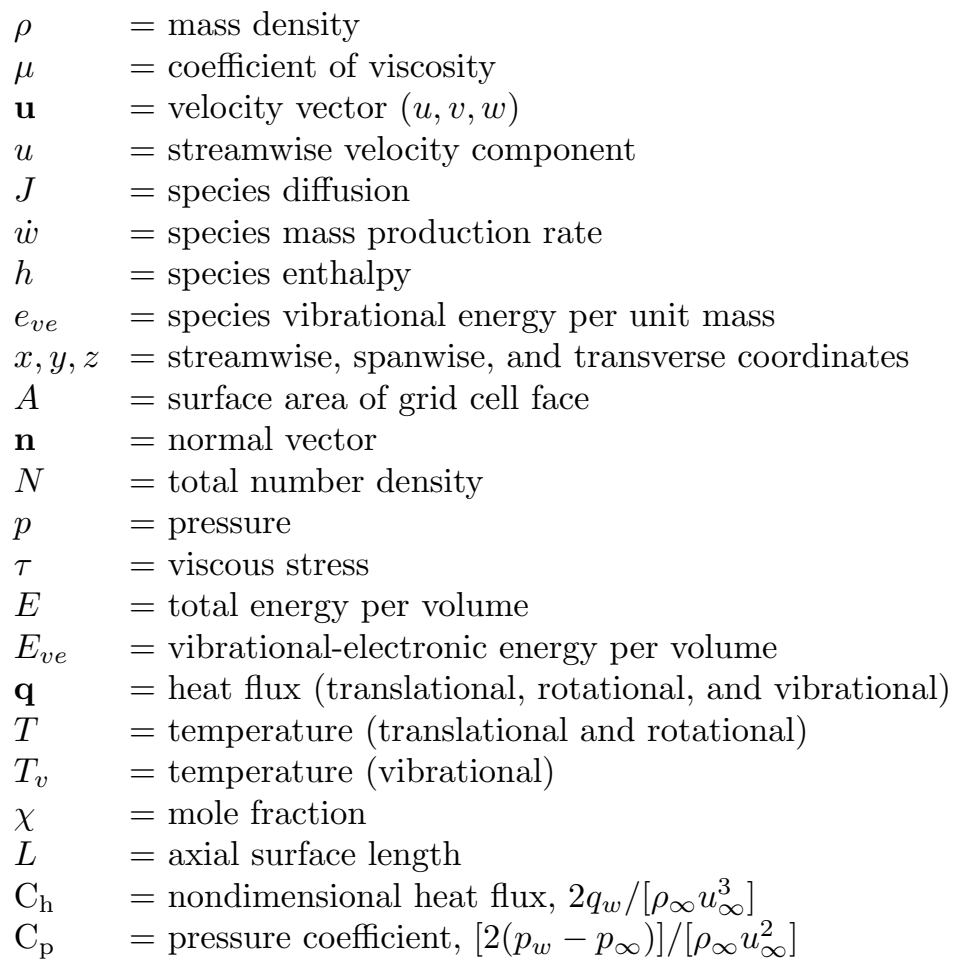

*Graduate Student, Student Member AIAA

${ }^{\dagger}$ Professor, Associate Fellow AIAA

${ }^{\ddagger}$ Senior Aerospace Engineer, Associate Fellow AIAA

This material is declared a work of the U.S. Government and is not subject to copyright protection in the United States. 


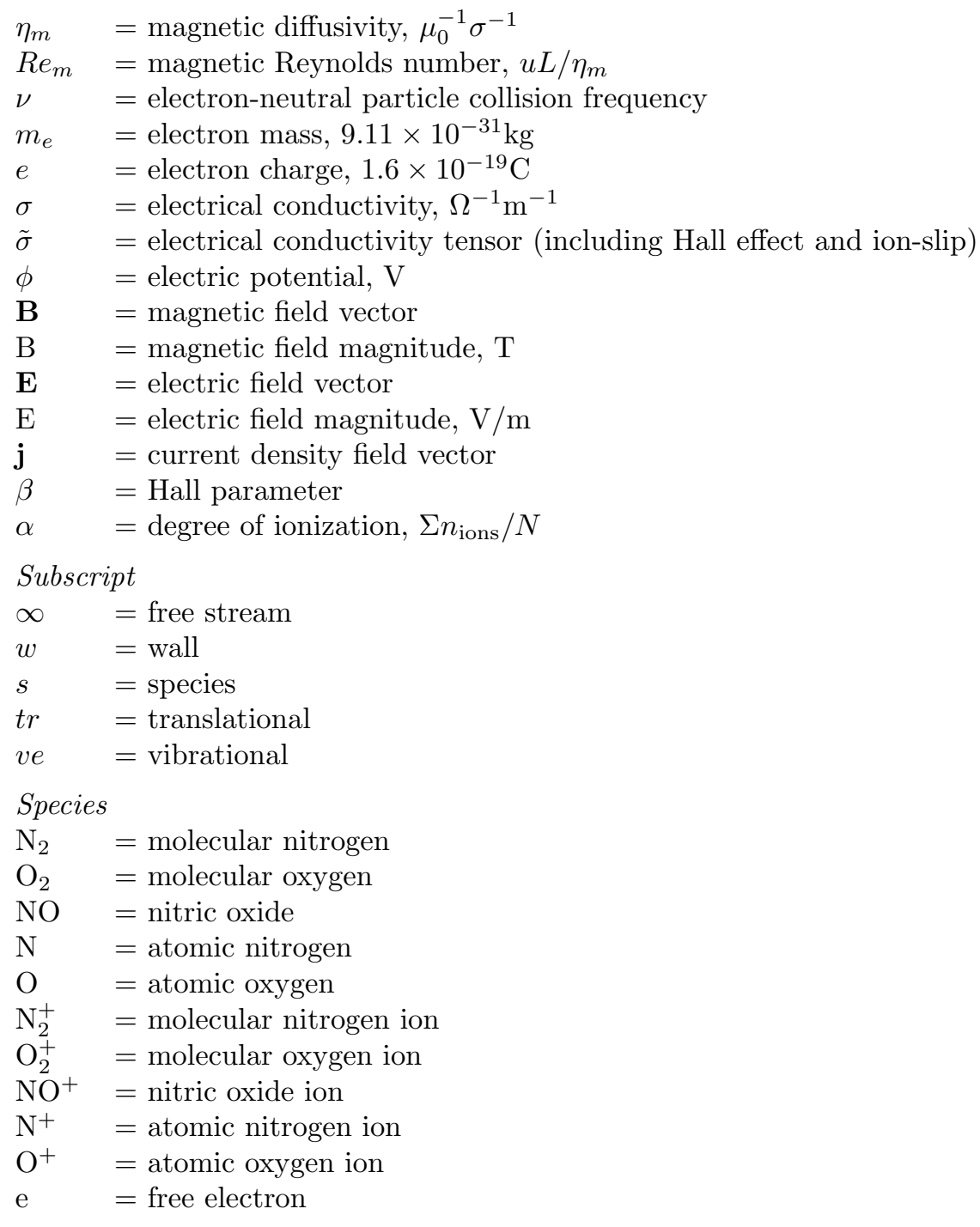

\section{Introduction}

Since the mid-1990's, plasma-assisted hypersonic flow control has experienced a resurgence of research interest. ${ }^{1-3}$ The revival has been credited to many factors including the expanding requirements for sustained hypersonic flight and rapid access to space. In addition, the numerous mechanical and material advances made during the past half century have given the area of flight-weight magnetoaerodynamics the rejuvenation necessary to warrant further exploration. One of the first to reevaluate the technology using modern CFD was Palmer, ${ }^{4}$ who performed first order spatially accurate simulations of the time-dependent Maxwell's equations, coupled to the Navier-Stokes equations to evaluate eleven species, chemically reacting air flow over a Mars return vehicle.

The rising costs for hypersonic experiments and the need for results within a greater range of flow-field conditions and increasing geometric complexity have continued to motivate the development of computational tools that are capable of accurately computing these plasma-assisted hypersonic flow control devices. This need has spurred numerous computational studies in the recent years, exploring all aspects of plasma-assisted flow enhancements, including flow control, ${ }^{5-10}$ local heat load mitigation, ${ }^{11-13}$ communications blackout, ${ }^{14,15}$ and magnetohydrodynamic (MHD) power extraction. ${ }^{16-18}$

Despite the large financial costs, limited facilities, and technical challenges, some recent experimental studies have been performed by Lineberry et al. ${ }^{19}$ Takizawa et al. ${ }^{20}$ Matsuda et al.,${ }^{21}$ and Gülhan et al. ${ }^{22}$ These efforts have provided additional validation cases for the ongoing computational pursuits. Of these 
experiments, the recent, partially released, experiment by Gülhan et al. will be of particular importance for future computational code validation as part of an an upcoming blind validation study.

A potential limitation of plasma-assisted devices is the large energy requirement necessary when they are employed to control large scale hypersonic flows by means of Joule heating through energy deposition. ${ }^{23}$ One possible way of improving the effectiveness and/or providing finer control is to utilize the ionized portion of the flow. The ionized flow can be subjected to electric and magnetic fields thereby producing additional/improved flow control. In order to simulate these effects, computational fluid codes need to be modified to accurately account for the MHD effects. This is accomplished by solving some form of Maxwell's equations. Although it is possible to solve Maxwell's equations directly coupled to the flow equations, ${ }^{24,25}$ most computational work in the area uses the current continuity equation in the low magnetic Reynolds number approximation, in a framework loosely coupled with the flow solver.

In the low magnetic Reynolds number approximation, the electrical conductivity appears in Ohm's law, which relates the current density field to the electric potential. Most research in computational hypersonic MHD bases the electrical conductivity on semi-empirical equations, which are valid for a particular range of temperatures, pressures, and species composition. While this approach is reasonable, it is not general, and is problematic when the flow conditions exceed the limited range of the approximation's validity. To this end, this paper explores the effects the electrical conductivity approximation has on the other flow properties by computing a solution for flow over a three-dimensional geometry with an embedded cathode (positive electrode) and anode (negative electrode). Three-dimensional calculations are performed. Barmin et al. ${ }^{26}$ have demonstrated the necessity of a three-dimensional solution of the MHD equations in order to prevent the introduction of unstable disturbances into the solution.

A three-dimensional parallelized MHD solver is developed, and iteratively coupled to a three-dimensional parallelized fluid solver to reduce the computational wall clock time. The new computational tool is used to estimate energy deposition into a flow by Joule heating, which is the result of an imposed potential difference across two electrodes embedded into a blunt leading edge geometry. The potential difference creates an arc discharge, which establishes current density and electric fields around the electrodes. These fields produce local energy deposition (Joule heating), in the flow, which can be employed for flow control.

\section{Method}

\section{A. Governing Equations}

Flow-field results are obtained using Computational Fluid Dynamics (CFD) to solve the Navier-Stokes equations. The CFD computations are executed using the Michigan Aerothermodynamic Navier-Stokes (LeMANS) code which was developed at the University of Michigan. ${ }^{27-29}$

LeMANS is a general 2D/axisymmetric/3D, parallel, unstructured finite-volume CFD code. The numerical fluxes between cells are discretized using a modified Steger-Warming Flux Vector Splitting (FVS) scheme, except near shock waves. In these regions the original Steger-Warming FVS scheme is used. LeMANS is able to employ a two-temperature or three-temperature model to account for thermal-nonequilibrium and a standard finite rate chemistry model for non-equilibrium chemistry. The two-temperature model assumes a single temperature $(T)$, which accounts for the translational and rotational energy modes of all species, while the vibrational energy mode is accounted for by a separate temperature $\left(T_{v}\right)$. In the three-temperature model, the rotational energy mode is broken out separately from the translational energy mode. ${ }^{30}$

The usual MHD conservation equations are solved with nonequilibrium air chemistry:

$$
\begin{gathered}
\frac{\partial \rho_{s}}{\partial t}+\nabla \cdot\left(\rho_{s} \mathbf{u}+J_{s}\right)=\dot{w}_{s} \\
\frac{\partial \rho \mathbf{u}}{\partial t}+\nabla \cdot(\rho \mathbf{u u}+p \mathbf{I}-\tau)=\mathbf{j} \times \mathbf{B} \\
\frac{\partial E}{\partial t}+\nabla \cdot\left((E+p) \mathbf{u}-\tau \cdot \mathbf{u}+\mathbf{q}+\Sigma\left(J_{s} h_{s}\right)\right)=\mathbf{j} \cdot \mathbf{E} \\
\frac{\partial E_{v e}}{\partial t}+\nabla \cdot\left(\left(E_{v e}\right) \mathbf{u}+q_{v e}+\Sigma\left(J_{s} e_{v, s}\right)\right)=\dot{w}_{v e}+\gamma(\mathbf{j} \cdot \mathbf{j}) / \sigma
\end{gathered}
$$


where (uu) in the conservation of momentum equation (2), is the $3 \times 3$ tensor containing all the products of the components of the velocity vector as seen in equation (5).

$$
\mathbf{u u}=\left[\begin{array}{ccc}
u^{2} & u v & u w \\
v u & v^{2} & v w \\
w u & w y & w^{2}
\end{array}\right]
$$

LeMANS assumes the fluid is continuous and Newtonian. It also assumes Stokes' hypothesis when determining the viscous stresses:

$$
\tau_{i j}=\mu\left(\frac{\partial u_{j}}{\partial x_{i}}+\frac{\partial u_{i}}{\partial x_{j}}\right)-\frac{2}{3} \mu \nabla \cdot \mathbf{u} \delta_{i j}
$$

Previous work considered a phenomenological model of dissipative heating to account for a thermal actuator. ${ }^{23}$ This was represented as an additional source term on the right side of the total energy equation (3), but is replaced here with Joule heating $(\mathbf{j} \cdot \mathbf{E})$. The conservation of momentum equation is modified to include the magnetic force $(\mathbf{j} \times \mathbf{B})$ on the right hand side of equation (2). The vibrational energy equation (4) is also modified with the inclusion of a Joule heating term $(\gamma \mathbf{j} \cdot \mathbf{j} / \sigma)$, where $(\gamma)$ is a constant that partitions electromagnetic energy deposition between different nonequilibrium modes $(\gamma \in[0,1])$. These additions constitute the effects the electric, current density, and magnetic fields have on the flow.

The vibrational energy source term $\left(\dot{w}_{v e}\right)$ is an approximation to the vibrational-electronic work done by the production/destruction of species due to chemistry, energy transfer between non-equilibrium modes, and work done by electrons by the electric field induced by the electron pressure gradient. Full details on the fluid solver including the chemistry and transport approximations, are available in Ref. 31.

For the remainder of the paper the two temperature energy model (nonequilibrium) is used and referred to as such $(\gamma=1)$. The simulations are performed using second-order accurate spatial discretization and carry double precision arithmetic throughout. This work assumes a standard finite-rate chemistry model for eleven species reacting air $\left(\mathrm{N}_{2}, \mathrm{O}_{2}, \mathrm{NO}, \mathrm{N}, \mathrm{O}, \mathrm{N}_{2}^{+}, \mathrm{O}_{2}^{+}, \mathrm{NO}^{+}, \mathrm{N}^{+}, \mathrm{O}^{+}\right.$, and e), where (e) represents the electrons. At present, force diffusion (drift) of the charge particles is neglected, except in Ohm's law.

\section{B. Low Magnetic Reynolds Number Approximation}

The three additional variables appearing in the conservation equations $(\mathbf{j}, \mathbf{B}, \mathbf{E})$ are determined by first noting that the magnetic Reynolds number, Eq. (6), is small for the cases of interest.

$$
R e_{m}=\frac{u L}{\eta_{m}}
$$

Consequently, it can be shown that the induced magnetic field can be neglected. ${ }^{32}$ This means only external magnetic fields are present in the flow (and must be specified). With the magnetic field assumed constant because of the low magnetic Reynolds number, a tensor form of the generalized Ohm's law, equation (7), is employed to obtain the current density $\mathbf{j}$.

$$
\mathbf{j}=\tilde{\sigma} \cdot(\mathbf{E}+\mathbf{u} \times \mathbf{B})
$$

The electrical conductivity tensor $(\tilde{\sigma})$ appearing in Ohm's law is a compact way of accounting for the Hall effect and ion slip as outlined in Refs. 33,34 and is described in detail in the following section. Following previous work, ${ }^{35} \mathrm{Eq}$. (7) is written in terms of the electric potential $(\phi)$ where $\mathbf{E}=-\nabla \phi$. Using current continuity $(\nabla \cdot \mathbf{j}=0)$, the final equation has the form of a Poisson equation, as seen in Eq. (8).

$$
\nabla \cdot \tilde{\sigma} \cdot[-\nabla \phi+\mathbf{u} \times \mathbf{B}]=0
$$

The electric potential is determined by using an explicit finite-volume method and appropriate boundary conditions as outlined in a later section. The electric field $(\mathbf{E}=-\nabla \phi)$ is computed directly from the electric potential solution following an approach for unstructured grids developed by Jawahar and Kamath. ${ }^{36}$ Full details of the derivation and assumptions are available in Ref. 35. 


\section{Hall Effect and Ion Slip}

As seen in equation (7), the computer code incorporates the tensor nature of the electrical conductivity $(\tilde{\sigma})$, which follows the outline in Refs. 33, 34, and is a compact way of accounting for ion-slip and the Hall effect. In this form, the Hall effect is accounted for in the electrical conductivity tensor, in Cartesian coordinates, as:

$$
\tilde{\sigma}=\frac{\sigma}{\mathrm{B}^{2}\left(1+\beta^{2}\right)}\left[\begin{array}{ccc}
\mathrm{B}^{2}+\beta^{2} \mathrm{~B}_{x}^{2} & \beta\left(\beta \mathrm{B}_{x} \mathrm{~B}_{y}-\mathrm{BB}_{z}\right) & \beta\left(\beta \mathrm{B}_{x} \mathrm{~B}_{z}+\mathrm{BB}_{y}\right) \\
\beta\left(\beta \mathrm{B}_{y} \mathrm{~B}_{x}+\mathrm{BB}_{z}\right) & \mathrm{B}^{2}+\beta^{2} \mathrm{~B}_{y}^{2} & \beta\left(\beta \mathrm{B}_{y} \mathrm{~B}_{z}-\mathrm{BB}_{z}\right) \\
\beta\left(\beta \mathrm{B}_{z} \mathrm{~B}_{x}-\mathrm{BB}_{y}\right) & \beta\left(\beta \mathrm{B}_{z} \mathrm{~B}_{y}+\mathrm{BB}_{x}\right) & \mathrm{B}^{2}+\beta^{2} \mathrm{~B}_{z}^{2}
\end{array}\right]
$$

where the Hall parameter $(\beta)$ is a function of the electron-neutral particle collision frequency $(\nu)$. The collision frequency is determined from the electrical conductivity as seen in equation (9).

$$
\begin{gathered}
\nu=\frac{q^{2} n_{e}}{m_{e} \sigma} \\
\beta=\frac{q \mathrm{~B}}{m_{e} \nu}=\frac{\sigma \mathrm{B}}{q n_{e}}
\end{gathered}
$$

Using this definition, the Hall parameter $(\beta)$ is computed as a function of the electrical conductivity $(\sigma)$, as seen in equation (10). The remaining simulations do not impose a magnetic field $(\mathbf{B}=0)$, so the electrical conductivity reverts to a scalar form.

\section{MHD Boundary Conditions}

Mixed boundary conditions are generally employed at a solid surface. For electrodes that are good conductors, the electric potential is specified, either as a fixed value or determined by auxiliary equations representing an external circuit. For an insulated boundary, the normal component of current is set to zero: $\mathbf{j} \cdot \mathbf{n}=\tilde{\sigma} \cdot(\mathbf{E}+\mathbf{u} \times \mathbf{B}) \cdot \mathbf{n}=0$. This can be a complicated boundary condition in the general case of tensor conductivity, but in the case of scalar conductivity and assuming no-slip wall conditions $(\mathbf{u}=0)$, it reduces to a vanishing normal electric field boundary condition $(\mathbf{E} \cdot \mathbf{n}=0)$.

The outward direction of a symmetry plane must also have a zero electric field $(\mathbf{E} \cdot \mathbf{n}=0)$. By definition the ghost cell center lies on the outward normal vector, so the symmetry plane boundary condition is: $\nabla \phi \cdot \mathbf{n}=\partial \phi / \partial n=0$. The proper boundary conditions in the far-field are less clear for aerodynamic MHD problems. For high accuracy, it may be necessary to solve the current continuity equation on a larger domain than the fluid conservation laws since the magnetic field can interact with the far-field. However, for external flows, the electrical conductivity should decay to a negligible value far from the body, so it is reasonable to set the normal component of the electric field to zero. The normal component of the electric field is assumed to be very small at the inlet $(\mathbf{E} \cdot \mathbf{n}=0)$. Finally, the outlet is assumed to be sufficiently downstream of the primary MHD interaction such that it is reasonable to set the normal component of the electric field to zero. Table 1 lists all domain boundaries and their respective conditions.

Table 1. Boundary conditions for MHD solver

\begin{tabular}{ccc}
\hline \hline Location & Type & Condition \\
\hline Inlet & Neumann & $\mathbf{E} \cdot \mathbf{n}=0$ \\
Far-field & Neumann & $\mathbf{E} \cdot \mathbf{n}=0$ \\
Symmetry & Neumann & $\mathbf{E} \cdot \mathbf{n}=0$ \\
Outlet & Neumann & $\mathbf{E} \cdot \mathbf{n}=0$ \\
Wall (electrode) & Dirichlet & $\phi=$ specified \\
Wall (insulating) & Neumann & $\mathbf{j} \cdot \mathbf{n}=0$ \\
\hline \hline
\end{tabular}

Dirichlet conditions are imposed in the ghost cells adjoining the wall (electrode) boundary such that the wall face electric potential equals the specified value. Neumann boundary conditions require the electric 
potential $(\phi)$ in the ghost cell to be determined iteratively in conjunction with the interiors cells of the MHD solver domain to satisfy a zero gradient electric field $(\mathbf{E} \cdot \mathbf{n}=\partial \phi / \partial n=0)$.

\section{MHD Parallelization and Validation}

The solution to the electric potential $(\phi)$ for a given flow iteration is determined within the MHD routine using a finite-volume method that iterates to a steady solution using an explicit Successive OverRelaxation (SOR) iterative technique. The SOR technique is an iterative explicit solver that utilizes the direction of change to the solution to extrapolate an improved solution based on a relaxation constant. The method converges more slowly than an implicit scheme, ${ }^{37}$ but is easier to implement and parallelize. The implemention of the MHD routine was previously validated for an analytic case with Dirichlet boundary conditions and for flow between two flat plates, and second-order accuracy was demonstrated. ${ }^{35}$

Parallelization of the MHD routine is accomplished using the framework already existing in the flow solver, LeMANS. LeMANS uses METIS ${ }^{38}$ to partition the domain amongst the processors, and utilizes MPI calls to pass ghost cell properties between processors. By using the existing framework, the computational effort to create new partitions, new ghost cells, and their corresponding boundary cell links between partitions, is retained. The drawback is that the MHD routine must use the same partitions, and subsequently, the same mesh as the flow solver. This can be problematic for specific geometries, especially when the grid resolution needed to solve a general form of Ohm's law is higher than that needed by the flow solver (e.g., regions of the domain where the electromagnetic field, but not the flow, has large gradients). This approach is still suitable because the computational cost per cell is equal in the MHD routine, and since METIS partitions the mesh to minimize the number of boundary cells and equalize the number of cells per partition, parallel efficiency of the MHD routine is consistent with LeMANS.

\section{Flow Between Parallel Electrodes}

Validation of the parallelized MHD module is accomplished by utilizing a previous validation exercise of flow between two parallel electrodes. ${ }^{35}$ This validation case was originally presented in the computational work by Gaitonde and Poggie. ${ }^{33}$ The two electrodes have a specified potential, so the top electrode plate $(z=1)$, is equal to one and the bottom plate $(z=0)$, is set to zero $\left(\phi_{\text {top }}=1, \phi_{\text {bottom }}=0\right)$. Neumann boundary conditions are employed along the remaining sides of the domain so the normal component of the gradient is zero $(\partial \phi / \partial n=0)$. Figure 1 illustrates the domain with a rectilinear nonuniform mesh used in the serial validation exercise detailed in Ref. 35. Cell clustering is applied near both electrodes using a bi-exponential decay of cell size along the $z$ axis, and uniform spacing along the electrode plate surface $(x$, and $y$ axes).

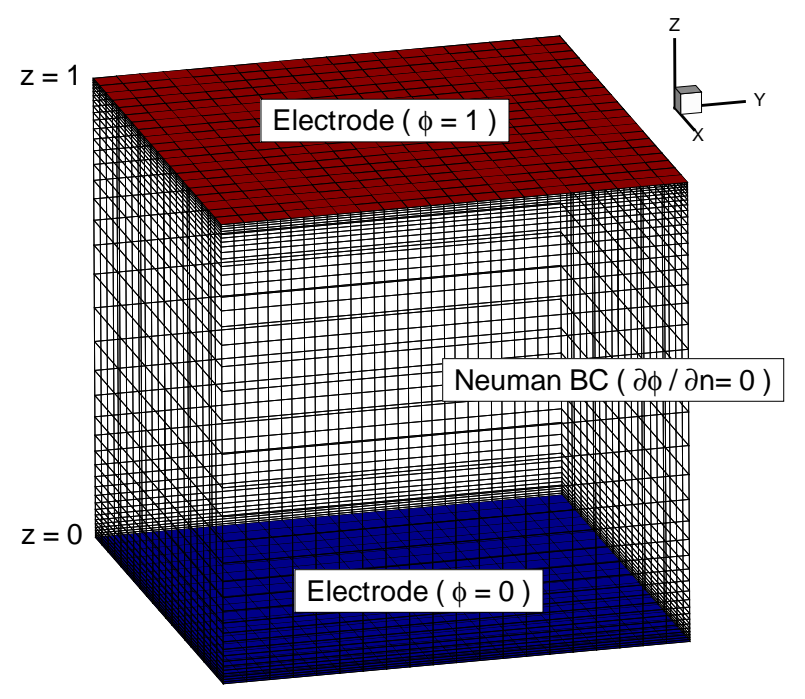

Figure 1. Nonuniform mesh $(20 \times 20 \times 40)$ for current flow between parallel electrode plates. 
For constant electrical conductivity $(\sigma=1)$, the theoretical solution reduces to a constant electric field. The resulting electric potential is: $\phi=z$ for the given boundary conditions. Figure 2 plots the computed solution obtained using four processors and the theoretical solution for constant electrical conductivity, with part of the solution domain cut away to reveal the interior cells. The computed solution matches the theoretical value and helps to verify that the MHD module is successfully parallelized.

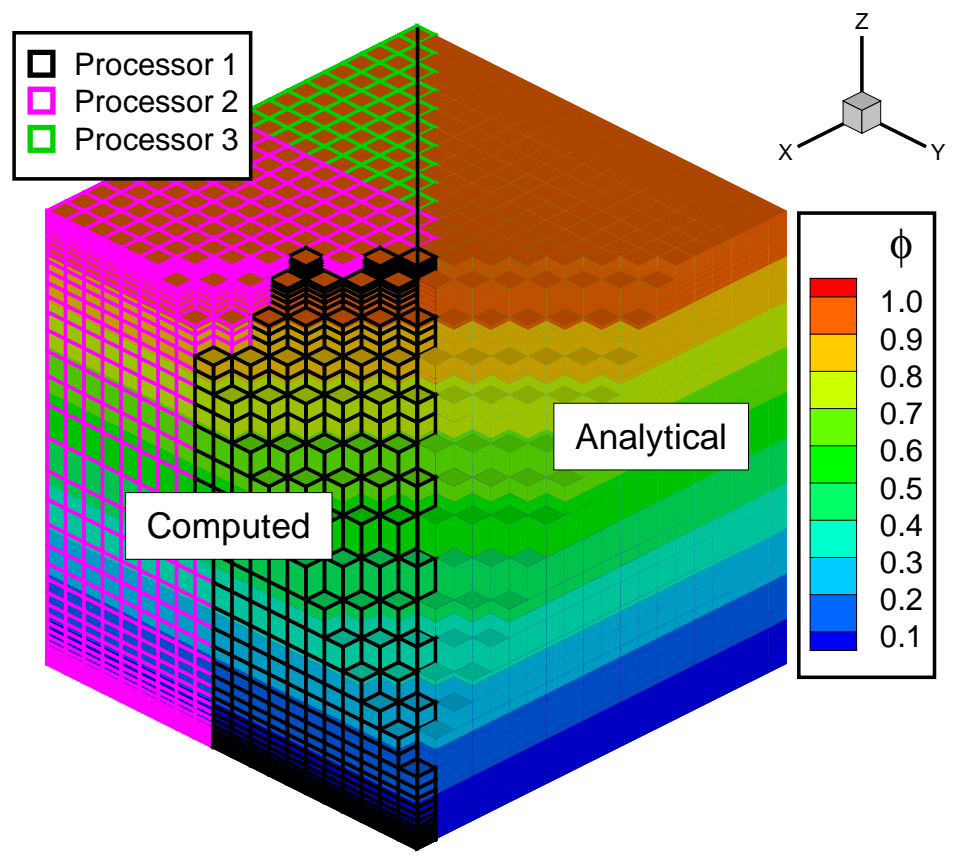

Figure 2. Electric potential contours between parallel electrode plates $(\sigma=1$ and $\mathbf{u}=0)$. The left side of the domain is composed of computed values using four processors (three shown), while the right side is the theoretical result. $(20 \times 20 \times 40$ mesh $)$

\section{Parallel Efficiency}

The parallel efficiency of LeMANS, with and without the MHD routine, is computed by simulating three-dimensional flow over a blunt leading edge, as seen in Fig. 3. The geometry has a length of $0.1 \mathrm{~m}$ and a vertical displacement of $0.05 \mathrm{~m}$. The body geometry follows a power-law-shaped profile $\left(z=x^{0.5}\right)$ and has an aerodynamic performance similar to a blunt body, ${ }^{39}$ which is beneficial for this investigation because a strong bow shock forms at the leading edge, which creates a minor concentration of ions in an otherwise neutral flow. The simulations are run using eleven species chemically reacting air, representative of conditions found at $40 \mathrm{~km}$. Table 2 lists the freestream conditions used.

A grid independence study is performed on the geometry using the chemically reacting, nonequilibrium flow solver. A structured grid is generated because the MHD routine cannot yet accommodate fully unstructured grids, and because a structured mesh is known to produce better results in regions near the surface of the body and through a shock. ${ }^{40}$ Exponential spacing is employed along each direction (along the body and radial from the body) so grid clustering occurs near the stagnation region. Exponential spacing places additional points exactly between existing points as the grid is doubly refined. The coarse grid employs 30 points along the geometry, 15 points radial from the body, and 10 points along the width of the body $(30 \times 15 \times 10)$. The points along the width of the body are also clustered using exponential spacing so clustering occurs around the half-width. The medium and fine grids double the points along each direction (medium: $60 \times 30 \times 20$, fine: $120 \times 60 \times 40$ ). A very fine grid $(120 \times 90 \times 40)$, is also generated because of differences in heat flux at the stagnation point for the medium and fine grids. Figure 3 illustrates cell clustering for the medium sized grid.

The pressure coefficient, Eq. (11), and the nondimensional heat flux, Eq. (12), are computed along the stagnation line at the body centerline for the different grids. 
Table 2. Flow conditions for Mach 12.6 air flow at an altitude of $40 \mathrm{~km}$.

\begin{tabular}{crl}
\hline \hline Parameter & \multicolumn{2}{c}{ Value } \\
\hline Mach & 12.6 & \\
$u_{\infty}$ & $4000.0 \mathrm{~m} / \mathrm{s}$ \\
$\mathrm{T}_{\infty}$ & $250.0 \mathrm{~K}$ \\
$\mathrm{~T}_{w}$ & $1000.0 \mathrm{~K}$ \\
$\mathrm{~T}_{0}$ & $8300.0 \mathrm{~K}$ \\
$\mathrm{p}_{\infty}$ & $289.0 \mathrm{~Pa}$ \\
$\rho_{\infty}$ & $0.004 \mathrm{~kg} / \mathrm{m}^{3}$ \\
$\chi_{N_{2}}$ & 0.79 & \\
$\chi_{O_{2}}$ & 0.21 & \\
$\mu_{\infty}$ & $1.6 \times 10^{-5}$ & $\mathrm{~kg} / \mathrm{m} \cdot \mathrm{s}$ \\
$\operatorname{Re}_{L}$ & $3.0 \times 10^{6}$ & \\
\hline \hline
\end{tabular}

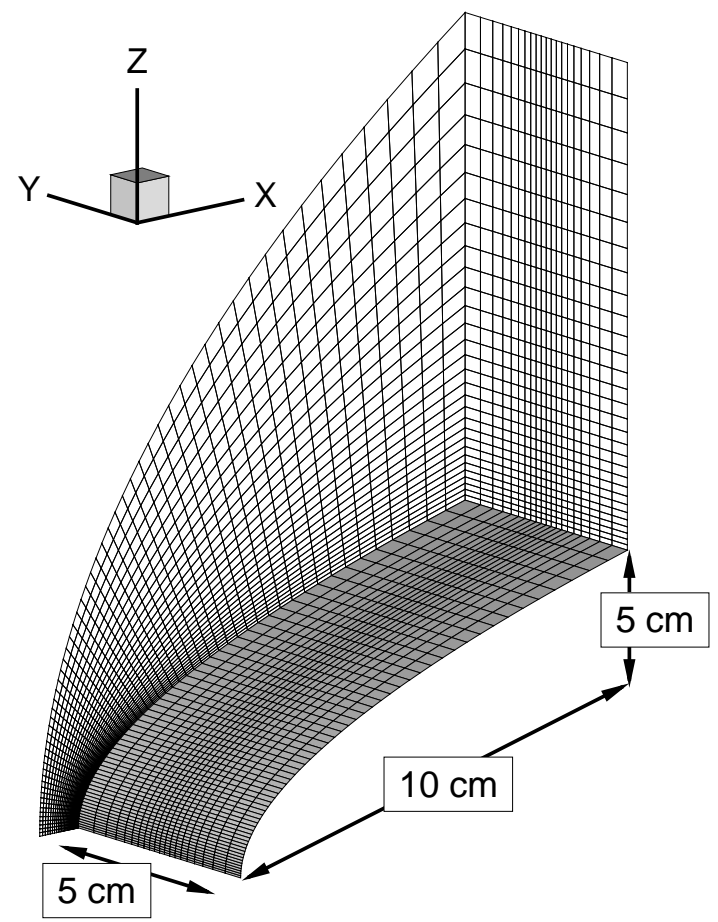

Figure 3. Geometry for a power-law shaped blunt leading edge $\left(z=x^{0.5}\right)$. 


$$
\begin{aligned}
\mathrm{C}_{\mathrm{p}} & =\frac{p_{w}-p_{\infty}}{1 / 2 \rho_{\infty} u_{\infty}^{2}} \\
\mathrm{C}_{\mathrm{h}} & =\frac{q_{w}}{1 / 2 \rho_{\infty} u_{\infty}^{3}}
\end{aligned}
$$

where $\left(q_{w}\right)$ is the heat flux to the wall. The heat flux to the wall is the summation of both the translationalrotational and vibrational-electronic heat fluxes $\left(q_{w}=q_{w t r}+q_{w v e}\right)$ for these nonequilibrium flows. Figure 4 plots the nondimensional heat flux and pressure coefficient for all four grids. The pressure coefficient is grid-independent for all the grids investigated.

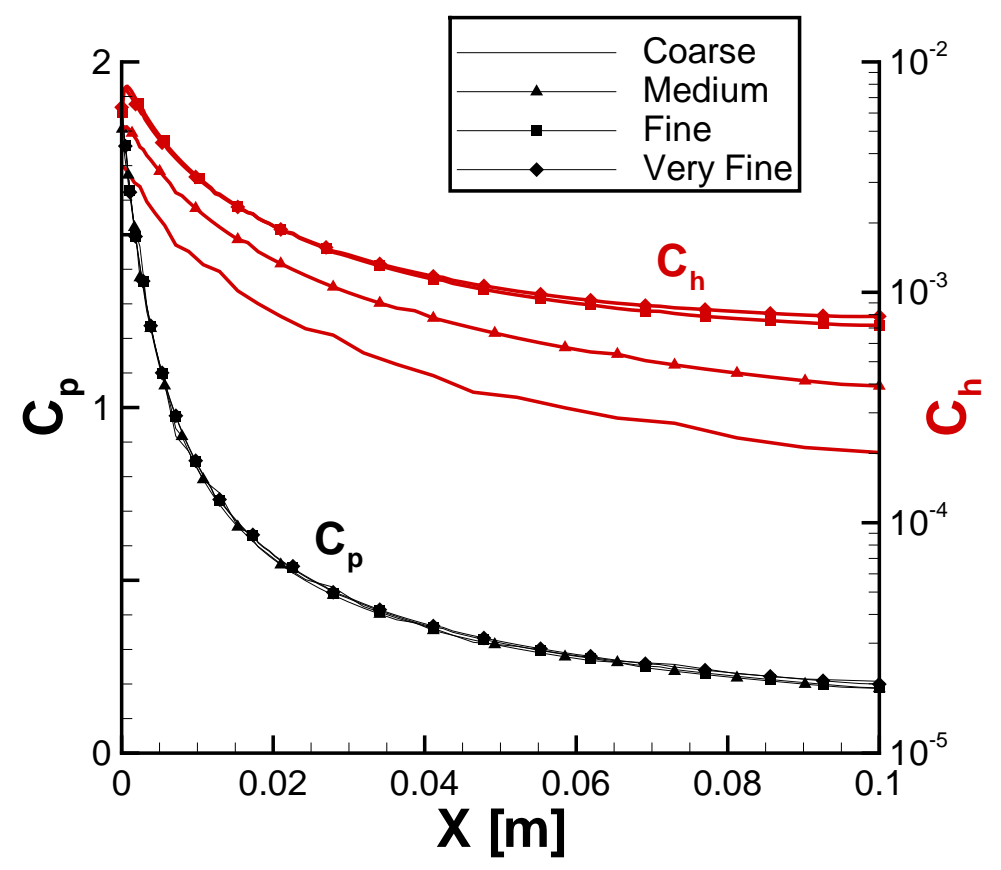

Figure 4. Coefficient of pressure and nondimensional heat flux along the half-width stagnation line of Mach 12.7 air over a power-law blunt leading edge for various grids. $(y=2.5 \mathrm{~cm})$

Excessively large cell spacing for the coarse grid in the stagnation region creates an artificially diffuse shock and an increase in the shock standoff distance, as observed in Fig. 5, which plots temperature contours for the coarse and fine grids. The difference in nondimensional heat flux between the medium and fine grids prompts the inclusion of a very fine grid, which increases the number of radial grid points, thereby improving grid resolution in the shock region. Similarity between the fine and very fine grids for the nondimensional heat flux is found to be acceptable, so the fine grid is assumed grid-independent for the fluid dynamics.

With a grid-independent mesh determined, parallel efficiency (speed up), is computed for the flow with and without the MHD routine. An arc discharge between an anode and cathode is simulated when the MHD routine is activated. The simulations are run for a specified number of iterations of the flow solver to determine the speed up as defined in equation (13).

$$
\text { speed up }=\frac{\text { serial time }}{\text { parallel time }}
$$

where (parallel time) is the wall time for the simulation (total CPU-hours / number of processors). Figure 6 plots the speedup versus the number of processors. The speedup with the MHD routine on is higher than with it off for part of the range because work-per-processor is higher relative to communication cost for the MHD case. Overall, the routine does not appear to affect the overall parallel efficiency of LeMANS. 


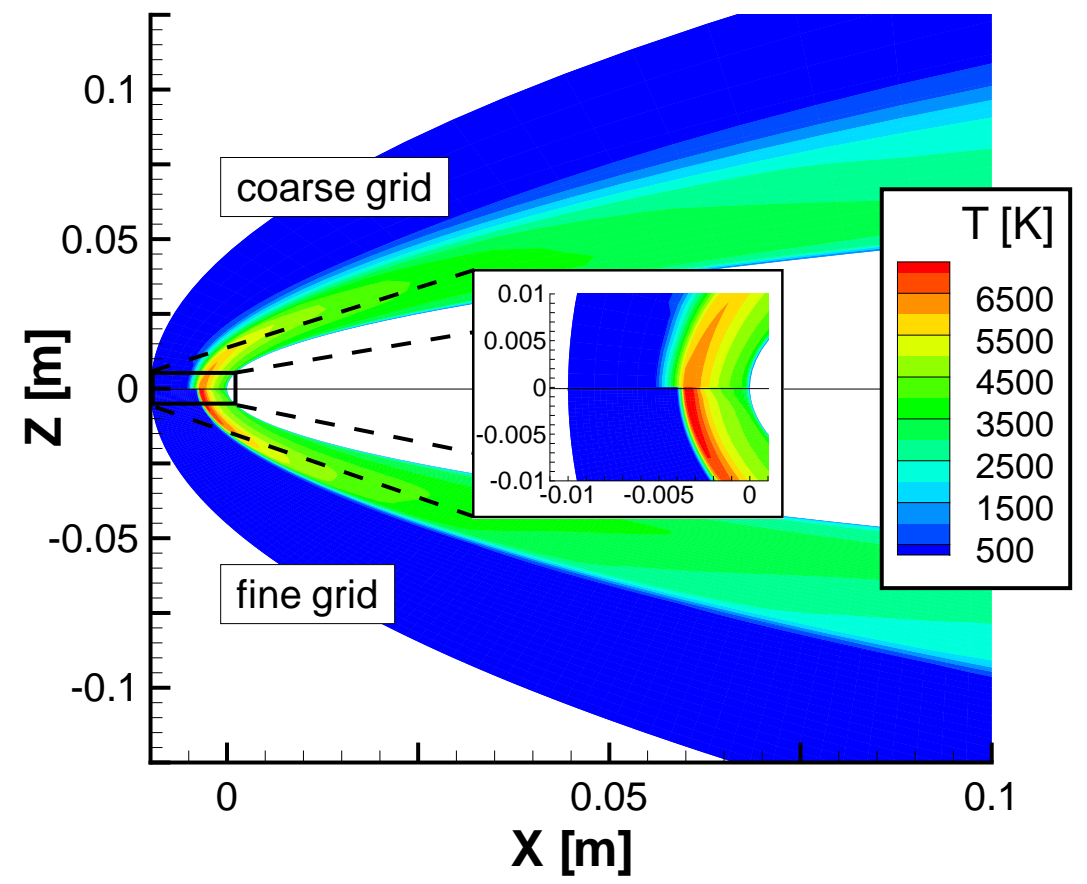

Figure 5. Temperature contours for the coarse $(30 \times 15 \times 10)$ and fine $(120 \times 60 \times 40)$ grids.

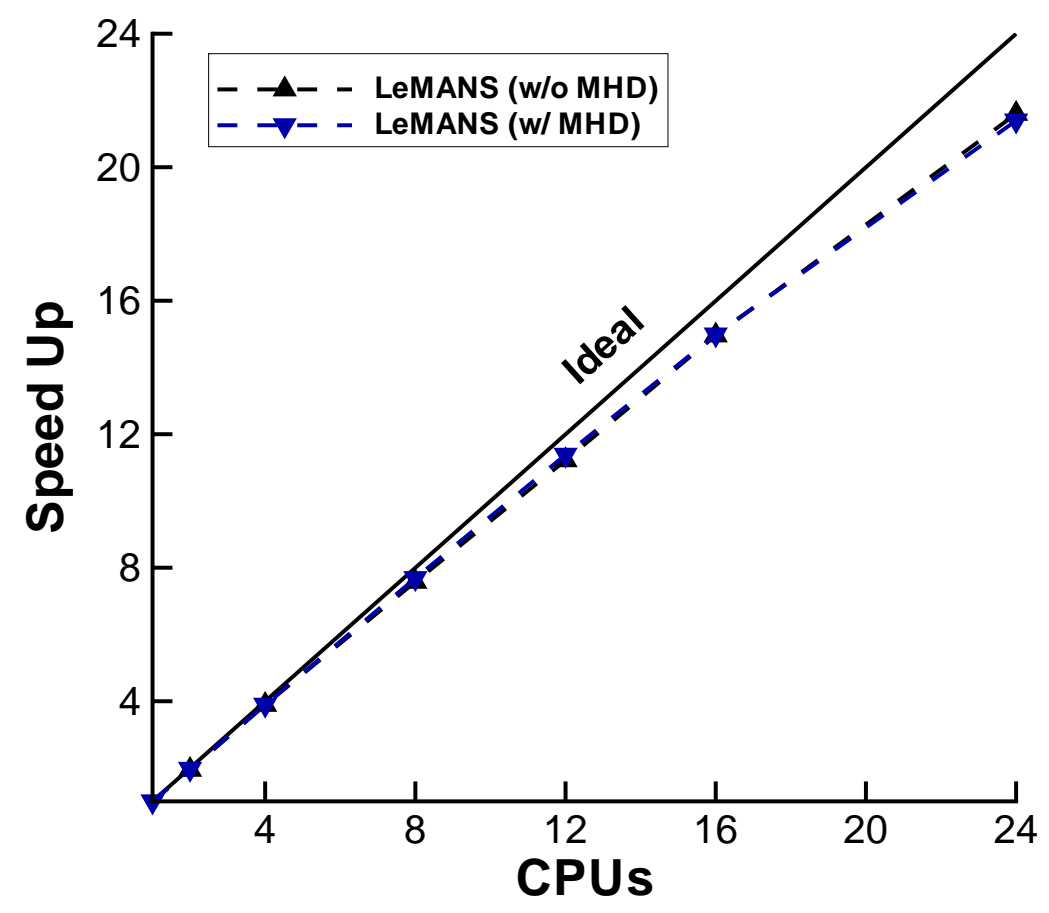

Figure 6. Speed up versus number of processors for LeMANS with and without the MHD routine. 


\section{Electrical Conductivity}

Since some of the first semi-analytic calculations demonstrating the potential benefits of plasma-assisted hypersonic flow control were computed by Resler and Sears ${ }^{41}$ in the late 1950's, the literature has mentioned improving the accuracy of these types of calculations by improving the electrical conductivity approximation employed. ${ }^{42}$ This section reviews two previously explored semi-analytic models ${ }^{35}$ and also investigates a surrogate model (response surface) of solutions to Boltzmann's equation.

\section{A. Semi-Analytic Models}

The first semi-analytic model explored was developed for weakly ionized flows by Chapman and Cowling, equation (14).

$$
\sigma=3.34 \times 10^{-10} \frac{\alpha}{Q T^{0.5}} \Omega^{-1} \mathrm{~m}^{-1}
$$

where $\alpha$ is the degree of ionization and $Q$ is the collision cross section. The degree of ionization is the summation of the ionized species number densities normalized by the total number density $\left(\alpha=\Sigma n_{\text {ions }} / N\right)$. The collision cross section is set to the vibrational cross-section for an ideal molecule $\left(Q=5 \times 10^{-13} \mathrm{~m}^{2}\right)$.

The second semi-analytic electrical conductivity model was developed by Raizer, ${ }^{43}$ and is only a function of temperature. His method relates the conductivity to an exponential function as seen in equation (15). This model is considered valid for air, nitrogen, and argon at $\mathrm{p}=1 \mathrm{~atm}$.

$$
\sigma=8300 \times e^{-36000 / T} \Omega^{-1} \mathrm{~m}^{-1}
$$

\section{B. Boltzmann Solver}

The semi-empirical models are compared with solutions to Boltzmann's equation using a solver developed by Weng and Kushner. ${ }^{44}$ This method of solving Boltzmann's equation is functionally equivalent to that proposed by Rockwood. ${ }^{45,46}$ Although the solver requires the translational temperature, pressure, and species mole fractions as input parameters, the solution to Boltzmann's equation only depends on the magnitude of the normalized electric field $(\mathrm{E} / N)$ and each species mole fraction $\left(\chi_{s}\right)$, except for the mole fraction of electrons since the solver assumes local charge neutrality. The solution is achieved using an extensive list of collision cross-section data taken from the compilations discussed in Refs. 47 and 48. The Boltzmann solver outputs the equilibrium transport coefficients which are used to compute the electrical conductivity for a range of $\mathrm{E} / N$.

Using the definitions of electron mobility and conductivity for a DC current, the electrical conductivity can be written in terms of the electron mobility $(\mu)$, the charge of an electron $\left(e=1.6 \times 10^{-19} \mathrm{C}\right)$, and the electron number density $\left(n_{e}\right)$, as given in Eq. (16).

$$
\sigma=\mu e n_{e}
$$

The electrical conductivity is computed for a given total number density $(N)$ by utilizing Eq. (17), which states the product of the electron mobility and total number density is a constant.

$$
\mu=\mu_{0}\left(\frac{N_{0}}{N}\right)
$$

This is combined with equation (16) to yield equation (18) which relates the electrical conductivity to the electron mobility (a transport coefficient found in the solutions to Boltzmann's equation), and the degree of ionization of the flow.

$$
\sigma=\mu_{0} N_{0} e\left(\frac{n_{e}}{N}\right) \Omega^{-1} \mathrm{~m}^{-1}
$$

A disadvantage of computing solutions to Boltzmann's equation is that the electrical conductivity $(\sigma)$ depends on the electric field $(\mathbf{E})$. Since the MHD solver depends on electrical conductivity $(\sigma)$ to determine the electric field (E), see equation (8), and the Boltzmann solver depends on the electric field (E) to determine

electrical conductivity $(\sigma)$, the Boltzmann solver must be iteratively coupled to the MHD solver in order to compute $\sigma, \mathbf{E}$, and $\mathbf{j}$ simultaneously, which increases the computational cost of the simulation. 


\section{Boltzmann Response Surface}

The main disadvantage of coupling the Boltzmann solver to the rest of the fluid solver is the large computational cost of using the Boltzmann solver directly. Using this approach, solutions to Boltzmann's equation must be computed for every cell, for every iteration. This is computationally prohibitive as it can take several CPU-minutes for the Boltzmann solver to find a single solution. One alternative approach is to create a surrogate model (response surface) of solutions to Boltzmann's equation for a large range of input conditions, and to use the response surface predictions instead of computing solutions to Boltzmann's equation directly.

The basic idea of surrogate modeling is to teach/develop a model by supplying a number of sample points which encompass the domain of interest (i.e. design space or design of experiment), and then evaluate its accuracy using additional test points. This work uses a surrogate modeling tool suite, SURROGATES Toolbox, developed by Goel and Viana ${ }^{49}$ which integrates several open-source tools thereby providing a general-purpose MATLAB ${ }^{\circledR}$ library of multidimensional function approximation methods.

The Polynomial Response Surface (PRS) is a commonly employed method which uses a polynomial function of degree $n$ to fit the data. The simplicity of the model makes it attractive and easy to implement, but it may require a high order polynomial to capture highly non-linear data sets. This work explores both third and fourth order PRS models.

As previously mentioned, the Boltzmann solver used for this work only depends on the magnitude of the electric field $(\mathrm{E} / N)$ and each species mole fraction $\left(\chi_{s}\right)$, which form the dimensions of the surrogate model. The eleven species air chemistry model $\left(\mathrm{N}_{2}, \mathrm{O}_{2}, \mathrm{NO}, \mathrm{N}, \mathrm{O}, \mathrm{N}_{2}^{+}, \mathrm{O}_{2}^{+}, \mathrm{NO}^{+}, \mathrm{N}^{+}, \mathrm{O}^{+}\right.$, and e) being employed in this work dictates that the number of dimensions for the surrogate responce model of electrical conductivity is eleven $\left(\mathrm{E} / N, \chi_{\mathrm{N}_{2}}, \chi_{\mathrm{O}_{2}}, \chi_{\mathrm{NO}}, \chi_{\mathrm{N}}, \chi_{\mathrm{O}}, \chi_{\mathrm{N}_{2}^{+}}, \chi_{\mathrm{O}_{2}^{+}}, \chi_{\mathrm{NO}^{+}}, \chi_{\mathrm{N}^{+}}, \chi_{\mathrm{O}^{+}}\right)$. The mole fraction of the electrons is unnecessary because the Boltzmann solver assumes charge neutrality. The design space dimension limits are: $\mathrm{E} / N \in[0.01,100 \mathrm{Td}], \chi_{\text {neutrals }} \in[0,100 \%]$, and $\chi_{\text {ions }} \in[0,1 \%]$ (townsend, $\mathrm{Td}=10^{-21} \mathrm{~V} \cdot \mathrm{m}^{2}$ ). The restriction on the ionic species is necessary because the present MHD formulation is only valid for weakly ionized flows (degree of ionization, $\alpha \leq 1 \%$ ). ${ }^{50}$

Sample locations are identified within the design space using a Latin Hypercube method which maximizes the minimum distance between dimensions of the sample points in order to reduce the correlation of the sample points generated. ${ }^{49}$ This work uses 4096 learning points ( 2 points per sub-domain for 11 dimensions) and 2977 testing points (1.45 points per sub-domain) to teach and evaluate the surrogate models (the number of sub-domains is $\left.2^{11}\right)$. Model accuracy is computed by comparing the computed test points $(\hat{h})$ with predictions from the model $(h)$ using the Mean Absolute Error (MAE), equation (19), and the Mean Absolute Percent Error (MAPE), equation (20).

$$
\begin{gathered}
\text { Mean Absolute Error (MAE) }=\frac{1}{n} \sum_{i=1}^{n}|\hat{h}-h|_{i} \\
\text { Mean Absolute Percent Error (MAPE) }=\frac{1}{n} \sum_{i=1}^{n}\left|\frac{\hat{h}-h}{(\hat{h}+h) / 2}\right|_{i}
\end{gathered}
$$

where the percent error is the normalized percent error to remove the bias when evaluating an overprediction. ${ }^{51}$ A summary of the two surrogate response surface models performance metrics are tabulated in Table 3 .

Table 3. Summary of surrogate model performance metrics.

\begin{tabular}{c|cccc}
\hline \hline Surrogate & MAPE & MAE & coefficients & conservativeness \\
\hline PRS $-3^{\text {rd }}$ order & $25.2 \%$ & 923 & 364 & $99.29 \%$ \\
PRS $-4^{\text {th }}$ order & $14.6 \%$ & 645 & 1365 & $99.40 \%$ \\
\hline \hline
\end{tabular}

The fourth order PRS model performs better across the design space, but is three times more computationally expensive because it requires fourth order coefficients. The MAE is the actual difference in electrical conductivity, which may be too large for regions of the design space where the electrical conductivity is small $\left(\sigma<1000 \Omega^{-1} \mathrm{~m}^{-1}\right)$. The model's conservativeness, the percent of the testing points predicting a positive response $(\sigma>0)$, is tabulated in Table 3, and is an important indicator of a suitable response surface model, 
because the model's predictions must be positive $(\sigma>0)$ to be physically plausible. A response model that is not conservative may require a cutoff parameter, which makes it less general, and consequently, less desirable.

\section{Electrical Conductivity Results}

To facilitate comparisons between the electrical conductivity models, the electrical conductivity is computed for flow over the power-law shaped blunt leading edge seen in Fig. 3 and freestream conditions listed in Table 2. The geometry is modified to include an embedded anode and cathode as seen in Fig. 7, and employs the coarse grid $(30 \times 15 \times 10)$.

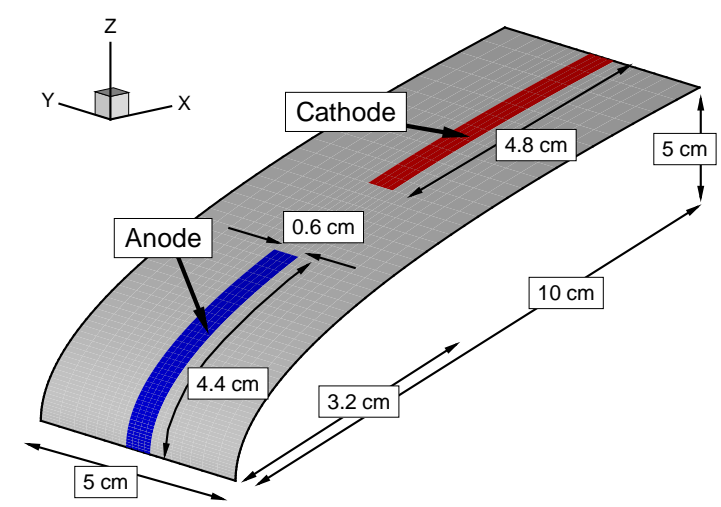

Figure 7. Dimensions of the embedded anode and cathode electrodes for the power-law shaped blunt leading edge geometry $\left(z=x^{0.5}\right)$.

The flow solver is run to steady-state without the MHD solver. The solver is then run for five additional flow iterations with the MHD solver iteratively coupled and a voltage potential between the anode and cathode of $100 \mathrm{~V}$. Raizer's electrical conductivity model is used to compute the electric potential for the first iteration of the Boltzmann response surface model because the response surface model depends on the magnitude of the electric field $(\mathrm{E} / N)$. The contours of the electrical conductivity, Fig. 8 illustrate the vast differences predicted by the models.

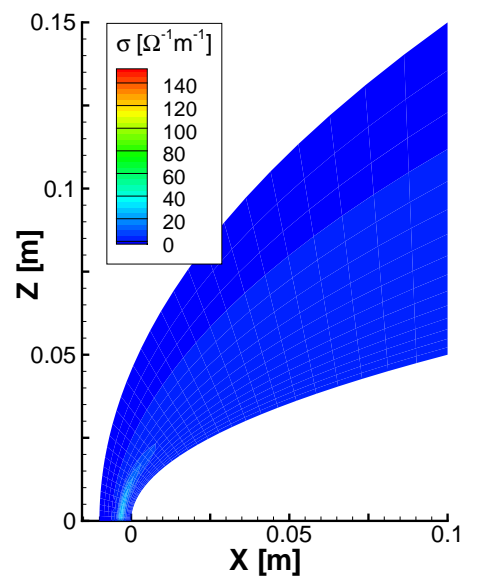

(a) Raizer

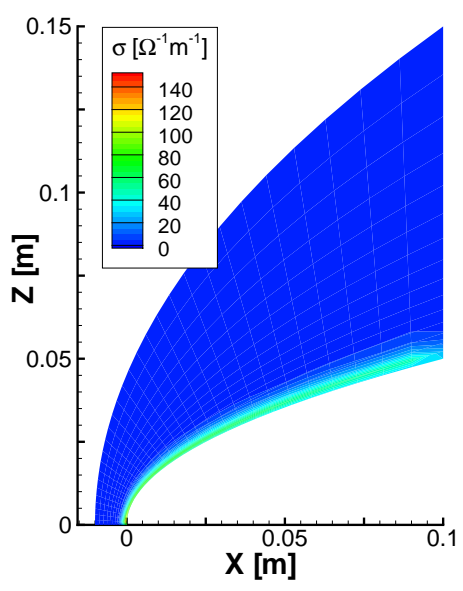

(b) Chapman and Cowling

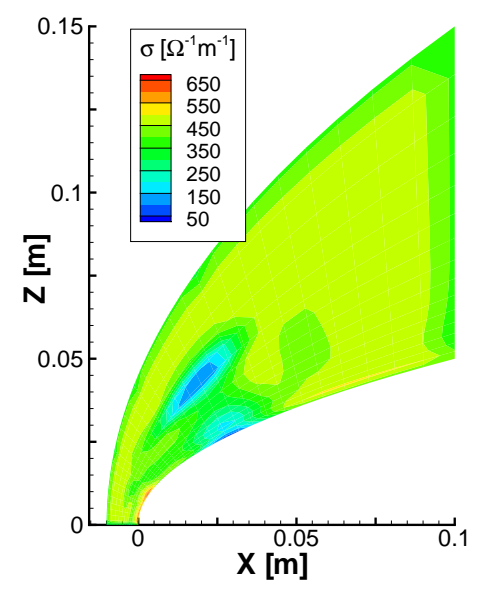

(c) Boltzmann Response Surface $\left(4^{\text {th }}\right.$ order PRS)

Figure 8. Electrical conductivity contours for various electrical conductivity models for Mach 12.7 air flow over a power-law shaped blunt leading edge with $100 \mathrm{~V}$ arc discharge $(y=2.5 \mathbf{c m}) .(30 \times 15 \times 10)$

Raizer's model only predicts significant electrical conductivity immediately downstream of the strong 
bow shock (where the temperature is the highest), whereas the Chapman and Cowling model predicts an increase of the conductivity near the surface (particularly in the stagnation region) because that part of the flow has the highest ionization fraction $\left(\alpha_{\max }=0.006\right)$. The $4^{\text {th }}$ order polynomial response surface predicts an increase in conductivity near the electrode surfaces, which is similar to the results to results from Chapmann and Cowling. However, the Boltzmann response surface model shows a significant decrease in conductivity in the region above an insulated wall. This decrease in electrical conductivity is due to the model's dependency on the magnitude of the electric field, which is low between the electrodes. Overall, the Boltzmann response surface model predicts much higher electrical conductivity then the two semi-analytic models, which is probably due to an insufficient approximation to solutions to Boltzmann's equation (poor modeling choice and/or a lack of learning points), and indicates further refinement of the surrogate modeling is needed before it can justifiably be used in the MHD routine. The remaining simulations will use Raizer's temperature-based conductivity model.

\section{Joule Heating}

In previous work, energy deposition into the flow was simulated by a phenomenological source term in the conservation of energy equation..$^{23}$ That work demonstrated that the level of flow control obtained was a function of the energy deposited. This section explores the amount of energy deposited into the flow via Joule heating ( $\mathbf{j} \cdot \mathbf{E})$ due to an arc discharge between two electrodes embedded in the geometry. The powerlaw shaped blunt leading edge geometry seen in Figures 3 and 7 is employed along with the freestream flow conditions listed in Table 2.

Simulations are run using the coarse grid $(30 \times 15 \times 10)$, and assume Raizer's electrical conductivity model. While these results are not grid independent, they provide reasonable estimates of the electric and current density field structures, and provide a reference for the expected amount of energy deposition into the flow due to an arc discharge between two electrodes. The anode is set to the imposed potential (negative), while the cathode is set slightly above zero volts. Figure 9 plots the current density contours in the $x$-direction along with the current lines.

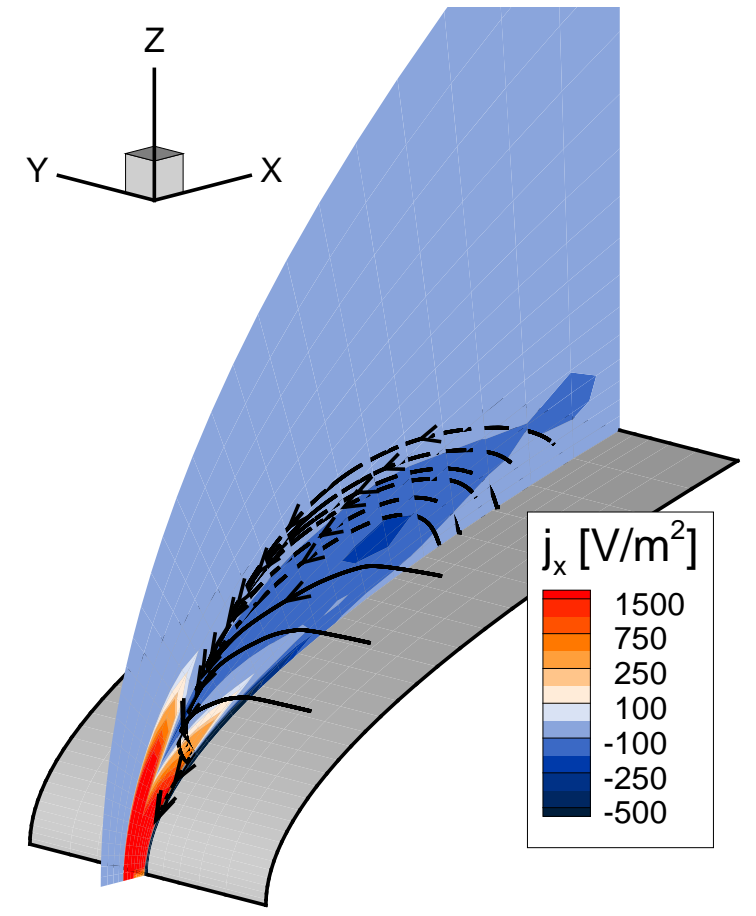

Figure 9. Current density contours in the $x$-direction and the current lines for flow between an anode electrode and cathode electrode embedded in a power-law shaped blunt leading edge geometry with an imposed potential of $100 \mathrm{~V}$. 
Current travels primarily from the cathode to anode, but some also comes from the symmetric boundaries $(y=0,5 \mathrm{~cm})$ to the anode along the high electrical conductivity 'rope' that exists transverse to the flow just downstream of the shock (due to the high post-shock temperature). Figure 10 plots Joule heating versus the potential difference between the anode and cathode. An increase in Joule heating is observed with an increase in voltage, and appears to increase exponentially. Grid independent results, however, are needed to strengthen any conclusions.

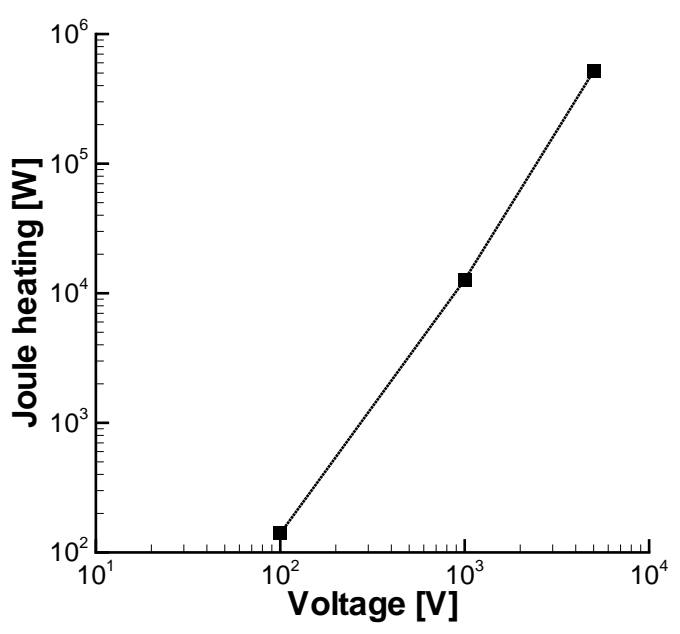

Figure 10. Total Joule heating due to an applied voltage between an anode and cathode embedded in a power-law shaped blunt leading edge geometry for various imposed voltages.

\section{Conclusions}

A three-dimensional parallelized MHD solver is developed and coupled to a three-dimensional fluid solver, LeMANS. Parallelization is accomplished using the parallel framework existing in LeMANS. Parallel efficiency of the MHD routine is measured, and found not to affect the overall parallel efficiency of LeMANS. The parallel MHD solver is also validated for flow between two electrodes and found to produce results consistent with theory and results obtained using a serial version of the routine.

A grid independence study is performed for flow over a three-dimensional power-law shaped blunt leading edge. The same geometry is used to explore electrical conductivity models by Raizer, Chapman and Cowling, and a new surrogate response surface of solutions to Boltzmann's equation. The response surface model demonstrates similarities to the other semi-analytic models but needs further refinement before being utilized in the standard MHD routine.

Joule heating versus voltage potential between two electrodes embedded in the power-law shaped blunt leading edge is computed on a relatively coarse grid, and is found to increase approximately exponentially as a function of the voltage. Grid independent results are needed to strengthen any conclusions.

\section{Future Work}

Continuation of this work includes a deeper exploration of various surrogate models in an effort to develop a more general response surface for solutions to Boltzmann's equation. In addition, the investigation of Joule heating due to an arc discharge will be continued to achieve grid independence, and will be expanded to investigate an arc discharge on a blunt elliptic cone.

In future studies, we will investigate how the MHD effects influence the species mass diffusion in the finite chemistry models. We will also explore the Hall effect and ion-slip. Of these, the Hall effect will be of particular importance as we study heat flux mitigation techniques using a magnet located within a hemisphere. 


\section{Acknowledgments}

The authors are indebted to the Michigan/AFRL/Boeing Collaborative Center in Aeronautical Sciences which provides funding to the first author. The first author would like to thank Mark Kushner for his valuable discussions on electrical conductivity and the use of his Boltzmann solver. The first author would also like to thank Pat Trizila for the numerous discussions on surrogate modeling and Felipe Viana for use of his surrogate modeling tool suite.

\section{References}

${ }^{1}$ Fomin, V. M., Tretyakov, P. K., and Taran, J.-P., "Flow Control using Various Plasma and Aerodynamic Approaches," Aerospace Science and Technology, Vol. 8, No. 5, July 2004, pp. 411-421.

${ }^{2}$ Shang, J. S., Surzhikov, S. T., Kimmel, R., Gaitonde, D., Menart, J., and Hayes, J., "Mechanisms of Plasma Actuators for Hypersonic Flow Control," Progress in Aerospace Sciences, Vol. 41, No. 8, November 2005, pp. 642-668.

${ }^{3}$ Bityurin, V., Bocharov, A., and Lineberry, J., "MHD Flow Control in Hypersonic Flight," 13th International Space Planes and Hypersonic Systems Technologies Conference, AIAA Paper 2005-3225, 2005.

${ }^{4}$ Palmer, G., "Magnetic Field Effects on the Computed Flow over a Mars Return Aerobrake," Journal of Thermophysics and Heat Transfer, Vol. 7, No. 2, April-June 1993, pp. 294-301.

${ }^{5}$ Bisek, N. J., Boyd, I. D., and Poggie, J., "Numerical Study of Energy Deposition Requirements for Aerodynamic Control of Hypersonic Vehicles," 46nd AIAA Aerospace Sciences Meeting and Exhibit, AIAA Paper 2008-1109, 2008.

${ }^{6}$ Kremeyer, K., Sebastian, K., and Shu, C.-W., "Computational Study of Shock Mitigation and Drag Reduction by Pulsed Energy Lines," AIAA Journal, Vol. 44, No. 8, August 2006, pp. 1720-1731.

7Yan, H. and Gaitonde, D., "Control of Edney IV Interaction by Energy Pulse," 44th AIAA Aerospace Sciences Meeting and Exhibit, AIAA Paper 2006-562, 2006.

${ }^{8}$ Menart, J., Stanfield, S., Shang, J., Kimmel, R., and Hayes, J., "Study of Plasma Electrode Arrangements for Optimum Lift in a Mach 5 Flow," 44th AIAA Aerospace Sciences Meeting and Exhibit, 2006, AIAA Paper 2006-1172.

${ }^{9}$ Girgis, I. G., Shneider, M. N., Macheret, S. O., Brown, G. L., and Miles, R. B., "Creation of Steering Moments in Supersonic Flow by Off-Axis Plasma Heat Addition," 40th AIAA Aerospace Sciences Meeting and Exhibit, 2002, AIAA Paper 2002-129.

${ }^{10}$ Gnemmi, P., Charon, R., Dupéroux, J.-P., and George, A., "Feasibility Study for Steering a Supersonic Projectile by a Plasma Actuator," AIAA Journal, Vol. 46, No. 6, June 2008, pp. 1308-1317.

${ }^{11}$ Bityurin, V. A., Vatazhin, A. B., and Gus'kov, O. V., "Hypersonic Flow Past the Spherical Nose of a Body in the Presence of a Magnetic Field," Fluid Dynamics, Vol. 39, No. 4, July 2004, pp. 657-666.

${ }^{12}$ Miles, R. B., Macheret, S. O., Shneider, M. N., Steeves, C., Murray, R. C., Smith, T., and Zaidi, S. H., "PlasmaEnhanced Hypersonic Performance Enabled by MHD Power Extraction," 43th AIAA Aerospace Sciences Meeting and Exhibit, AIAA Paper 2005-561, 2005.

${ }^{13}$ Katsurayama, H., Kawamura, M., Matsuda, A., and T., A., "Kinetic and Continuum Simulations of Electromagnetic Control of a Simulated Reentry Flow," Journal of Spacecraft and Rockets, Vol. 45, No. 2, March-April 2008, pp. $248-254$.

${ }^{14}$ Hodara, H., "The Use of Magnetic Fields in the Elimination of the Re-Entry Radio Blackout," Proceedings of the IRE, Vol. 4, No. 12, December 1961, pp. 1825-1830.

${ }^{15}$ Kim, M., Keidar, M., and Boyd, I. D., "Analysis of an Electromagnetic Mitigation Scheme for Reentry Telemetry Through Plasma," Journal of Spacecraft and Rockets, Vol. 45, No. 6, November-December 2008, pp. 1223-1229.

${ }^{16}$ Macheret, S. O., Shneider, M. N., and Candler, G. V., "Modeling of MHD Power Generation on Board Reentry Vehicles," 42nd AIAA Aerospace Sciences Meeting, AIAA Paper 2004-1024, 2004.

${ }^{17}$ Wan, T., Suzuki, R., Candler, G., Macheret, S., and Schneider, M., "Three Dimensional Simulation of Electric Field and MHD Power Generation During Re-Entry," 36th AIAA Plasmadynamics and Lasers Conference, AIAA Paper 2005-5045, 2005.

${ }^{18}$ Fujino, T., Yoshino, T., and Ishikawa, M., "Prediction of Generator Performance and Aerodynamic Heating of Reentry Vehicle Equipped with On-board Surface Hall Type MHD Generator," 39th Plasmadynamics and Lasers Conference, AIAA Paper 2008-4225, 2008.

${ }^{19}$ Linberry, J. T., Bityurin, V. A., and Vatazhin, A. B., "Cylinder with Current in Hypersonic Flow," Proc. 3rd Workshop on Magneto-Plasma Aerodynamics in Aerospace Applications, Institute of High Temperatures of RAS (IVTAN) (2001), pp. 15.

${ }^{20}$ Takizawa, Y., Sato, S., Abe, T., and Konigorski, D., "Electro-Magnetic Effect on Shock Layer Structure in ReentryRelated High-Enthalpy Flow," 35th AIAA Plasmadynamics and Lasers Conference, AIAA Paper 2004-2162, 2004.

${ }^{21}$ Matsuda, A., Kawamura, M., Takizawa, Y., Otsu, H., Konigorski, D., Sato, S., and Abe, T., "Experimental Investigation of the Hall Effect for the Interaction between the Weakly-Ionized Plasma Flow and Magnetic Body," 45th Aerospace Sciences Meeting, AIAA Paper 2007-1437, 2007.

${ }^{22}$ Gülhan, A., Esser, B., Koch, U., Siebe, F., Riehmer, J., Giordano, D., and Konigorski, D., "Experimental Verification of Heat-Flux Mitigation by Electromagnetic Fields in Partially-Ionized-Argon Flows," Journal of Spacecraft and Rockets, Vol. 46, No. 2, March-April 2009, pp. 274-283.

${ }^{23}$ Bisek, N. J., Boyd, I. D., and Poggie, J., "Numerical Study of Plasma-Assisted Aerodynamic Control for Hypersonic Vehicles," Journal of Spacecraft and Rockets, Vol. 46, No. 3, May-June 2009, 568-576.

${ }^{24}$ MacCormack, R., "Numerical Simulation of Aerodynamic Flow Including Induced Magnetic and Electric Fields," 39th Plasmadynamics and Lasers Conference, AIAA Paper 2008-4010, 2008.

${ }^{25}$ D'Ambrosio, D. and Giordano, D., "Two-Dimensional Numerical Methods in Electromagnetic Hypersonics Including Fully Coupled Maxwell Equations," 39th Plasmadynamics and Lasers Conference, AIAA Paper 2008-4013, 2008. 
${ }^{26}$ Barmin, A. A., Kulikovskiy, A. G., and Pogorelov, N. V., "Shock-Capturing Approach and Nonevolutionary Solutions in Magnetohydrodynamics," Journal of Computational Physics, Vol. 126, No. 1, June 1996, pp. 77-90.

${ }^{27}$ Scalabrin, L. C. and Boyd, I. D., "Development of an Unstructured Navier-Stokes Solver For Hypersonic Nonequilibrium Aerothermodynamics," 38th AIAA Thermophysics Conference, AIAA Paper 2005-5203, 2005.

${ }^{28}$ Scalabrin, L. C. and Boyd, I. D., "Numerical Simulation of Weakly Ionized Hypersonic Flow for Reentry Configurations," 9th AIAA/ASME Joint Thermophysics and Heat Transfer Conference, AIAA Paper 2006-3773, 2006.

${ }^{29}$ Scalabrin, L. C. and Boyd, I. D., "Numerical Simulation of the FIRE-II Convective and Radiative Heating Rates," 39th AIAA Thermophysics Conference, AIAA Paper 2007-4044, 2007.

${ }^{30}$ Holman, T. D. and Boyd, I. D., "Numerical Investigation of the Effects of Continuum Breakdown on Hypersonic Vehicle Surface Properties," 40th AIAA Thermophysics Conference, AIAA Paper 2008-3928, 2008.

${ }^{31}$ Scalabrin, L. C., "Numerical Simulation of Weakly Ionized Hypersonic Flow Over Reentry Capsules," Ph.D. Thesis, Univ. of Michigan, 2007.

${ }^{32}$ Shercliff, J., A Textbook of Magnetohydrodynamics, Pergamon Press, 1965.

${ }^{33}$ Gaitonde, D. V. and Poggie, J., "Elements of a Numerical Procedure for 3-D MGD Flow Control Analysis," 40th AIAA Aerospace Sciences Metting and Exhibit, AIAA Paper 2002-198, 2002.

${ }^{34}$ Gaitonde, D. V., "A High-Order Implicit Procedure for the 3-D Electric Field in Complex Magnetogasdynamic Simulations," Computers and Fluids, Vol. 33, No. 3, March 2004, pp. 345-374.

${ }^{35}$ Bisek, N. J., Boyd, I. D., and Poggie, J., "Numerical Study of Electromagnetic Aerodynamic Control of Hypersonic Vehicles," 47th AIAA Aerospace Sciences Meeting and Exhibit, AIAA Paper 2009-1000, 2009

${ }^{36}$ Jawahar, P. and Kamath, H., "A High-Resolution Procedure for Euler and Navier-Stokes Computations on Unstructured Grids," Journal of Computational Physics, Vol. 164, October 2000, pp. 164-203.

${ }^{37}$ Tannehill, J. C., Anderson, D. A., and Pletcher, R. H., Computational Fluid Mechanics and Heat Transfer, 2nd ed., Hemisphere Publishing Corporation, 1997.

${ }^{38}$ Karypis, G. and Kumar, V., "METIS: A Software Package for Partitioning Unstructured Graphs, Partitioning Meshes, and Computing Fill-Reducing Orderings of Sparse Matrices," University of Minnesota, MN, 1998.

${ }^{39}$ Mason, W. H. and Lee, J., "Aerodynamically Blunt and Sharp Bodies," 1992.

${ }^{40}$ Candler, G., "Unstructured Grid Approaches for Accurate Aeroheating Simulations," 18th AIAA Computational Fluid Dynamics Conference, AIAA Paper 2007-3959, 2007.

${ }^{41}$ Resler, E. L. and Sears, W. R., "The Prospects for Magneto-Aerodynamics," Journal of Aeronatuical Sciences, Vol. 25, April 1958, pp. 235-245, 258.

${ }^{42}$ Resler, E. L. and Sears, W. R., "The Prospects for Magneto-Aerodynamics Correction and Addition," Journal of Aero/Space Sciences, Vol. 26, No. 5, May 1959, pp. 319.

${ }^{43}$ Raizer, Y. P., Gas Discharge Physics, Springer-Verlag, 1991.

${ }^{44}$ Weng, Y. and Kushner, M. J., "Method for Including Electron-Electron Collisions in Monte Carlo Simulations of Electron Swarms in Partially Ionized Gases," Physical Review A, Vol. 42, No. 10, November 1990, pp. 6192-6200.

${ }^{45}$ Rockwood, S. D., "Elastic and Inelastic Cross Sections for Electron-Hg Scattering from Hg Transport Data," Physical Review A, Vol. 8, No. 5, November 1973, pp. 2348-2358.

${ }^{46}$ Rockwood, S. D., "Effect of Electron-Electron and Electron-Ion Collisions in $\mathrm{Hg}, \mathrm{CO}_{2} / \mathrm{N}_{2} / \mathrm{He}$, and $\mathrm{CO} / \mathrm{N}_{2}$ discharges," Journal of Applied Physics, Vol. 45, No. 12, December 1974, pp. 5229-5234.

${ }^{47}$ Dorai, R. and Kushner, M. J., "A Model for Plasma Modification of Polypropylene using Atmospheric Pressure Discharges," Journal of Physics D: Applied Physics, Vol. 36, No. 6, March 2003, pp. 666-685.

${ }^{48}$ Stafford, D. S. and Kushner, M. J., "O $\mathrm{O}_{2}\left({ }^{1} \Delta\right)$ Production in $\mathrm{He} / \mathrm{O}_{2}$ mixtures in Flowing Low Pressure Plasmas," Journal of Applied Physics, Vol. 96, No. 5, September 2004, pp. 2451-2465.

${ }^{49}$ Viana, F. A. C., SURROGATES Toolbox Users Guide, 2009, http://fchegury.googlepages.com.

${ }^{50}$ Cambel, A. B., Plasma Physics and Magnetofluid-Mechanics, McGraw-Hill, 1963, p. 171.

${ }^{51}$ Makridakis, S., "Accuracy Measures: Theoretical and Practical Concerns," International Journal of Forecasting, Vol. 9, No. 4, December 1993, pp. 527-529. 